\title{
PHYSICS AND CONTROL OF THE AFTERGLOW HCI-BEAM PULSEWIDTHS FOR SYNCHROTRON AND ATOMIC PHYSICS
}

\author{
M. Niimura, A. Chutjian, S. Smith, JPL/Caltech, Pasadena, CA 91109, USA \\ M. Lamoureaux, Univ. of P. \& M. Curie, 75252 Paris, France \\ A. Goto, Y. Yano, RIKEN, Saitama 351-0198, Japan
}

\begin{abstract}
Afterglow mode of operation in the ECR ion source (ECRIS) has found its applications not only in synchrotron accelerators but also in atomic physics, for it can provide a pulsed beam of highly charged ions (HCI) with much higher current than extractable at $\mathrm{cw}$ mode. However, control of pulsewidths and optimization of operation conditions are not a trivial matter without knowing relevant physics. This paper explains several important physics discovered through analysis of our and other's experimental data. Experimentally observable typical ion-pulse waveforms, $\mathrm{n}_{\mathrm{i}}(\mathrm{t})$, were successfully simulated, including the timings of hot-electron loss. Time-dependent depth of the ion trapping potential-well, $\Delta \phi(\mathrm{t})$, is only the information needed for the simulation of $n_{i}(t)$ at nonlinear mode. On the other hand, electron density $\left(\mathrm{n}_{\mathrm{e}}\right)$, electron temperature, and charge state $(\mathrm{Z})$ are needed for that of quiescent decay mode. Most importantly, this work has generated the evidences of the interchange instability (ICI), responsible particles for $\Delta \phi(\mathrm{t})$, presence of a hot-electron shell/ring, rotation of hot electrons, cold-electrons to stabilize ICI, and mass (M) to Z dependence of ICI-threshold. ICI theory predicts that a sharp-rising pulse is likely to be produced when $\mathrm{M} / \mathrm{Z}$ is smaller, because the unstable equilibrium condition $\left(\mathrm{n}_{\mathrm{h}} \geq \mathrm{n}_{\mathrm{c}}\right)$ is easily met before turning off the rfpower. Trailing edge of the pulse can be shortened by increasing the $\mathrm{Z}$ and $\mathrm{n}_{\mathrm{e}}$, but decreasing the rf-power.
\end{abstract}

\section{INTRODUCTION}

Some synchrotrons such as the large hadron collider (LHC) are demanding a very short pulse $(\leq 0.4 \mathrm{~ms})$ of HCIs at the frequency around $5 \mathrm{~Hz}$. Such an injector may be feasible from the ECRIS operating at an afterglow mode. Presently, however, its pulsewidth as well as pulse-shape are hardly controllable.

Although the beam intensity could be much less than those $\left(0.1 \sim 1\right.$ emA of $\left.\mathrm{Pb}^{27+}, 28+\right)$ required for $\mathrm{LHC}$ accelerator, pulsed $\mathrm{HCI}$ beams at an elevated intensity are quite useful in the field of atomic physics as well. In one of the examples, a pulsed HCI beam is injected into a Kingdon ion-trap, and oscillator strengths of the metastable states of HCIs are determined by measuring their lifetimes. Only a requirement here is to operate ECRIS at a high vacuum not to quench metastable ions.

Physics of the afterglow mode is not well understood, while its controlled operation is never straightforward. We therefore firstly investigate the physics to conform the complex time histories of an afterglow pulse.

\section{PHYSICS OF PULSEWIDTHS}

\subsection{Rise, Decay and Oscillatory Profiles}

Our experimental data have revealed that the profiles of afterglow pulses can be classified into two different modes. One is quiescent decay mode (QDM) and another non-linear mode (NLM), in accordance with the terminology of Geller [1]. We have not observed the third mode, turbulent or Bohm type fast diffusion. An afterglow waveform always ends by QDM with an exponential tail.

\subsection{Identification of the Type of Ion Diffusion}

The simplest or textbook-shown waveform of afterglow ion-beam pulse is probably the one to rise straight, followed immediately by an exponential(-like) decay. Such a decay should be resulted from a diffusion process of the test particles (ion), colliding against the field particles (electron and/or ion).

Suppose the HCIs diffuse parallel to the mirror magnetic field $\left(\mathrm{B}_{\mathrm{Z}}\right)$, then the corresponding diffusion coefficient is given by $D_{i} \equiv \mathrm{v}_{\mathrm{i}} / \mathrm{v}_{\mathrm{ie}}$, where $\mathrm{v}_{\mathrm{i}} \equiv\left(\kappa \mathrm{T}_{\mathrm{i}} / \mathrm{m}_{\mathrm{i}}\right)^{1 / 2}$ is the ion thermal velocity and $v_{\mathrm{ie}}=v_{\mathrm{ei}} \equiv \eta\left(\mathrm{n}_{\mathrm{e}} \mathrm{e}^{2 / \mathrm{m}_{\mathrm{e}}}\right)$ is the Coulomb collision frequency. Substitution of the Fick's law for diffusing ion flux, $\Gamma_{i}=-D_{i} \nabla n_{i}$, into the equation of continuity gives, during the afterglow phase when $S=0$ or rf-power source is turned off,

$$
\frac{\partial n_{i}}{\partial t}+\nabla \bullet \Gamma_{i}=\frac{\partial n_{i}}{\partial t}-D_{i} \nabla^{2} n_{i}=S=0
$$

If $D_{i}$ is independent from the density of test particles $\left(\mathrm{n}_{\mathrm{i}}\right)$, Eq. (1) can be solved easily to give the time dependent solution in an exponential form: $n_{i}=n_{p k} e^{-t / \tau}$. The $1 / e-$ decay time is then given by $\tau_{/ /}=(2 \mathrm{~L} / \pi)^{2 /} D_{i}$, where $2 \mathrm{~L}$ is the size in which HCIs of the charge state $\mathrm{Z}$ had been trapped. Using the well-known formula, $v_{e i}\left(s^{-1}\right)=2.0 \times 10^{-6} Z n_{e}\left(\mathrm{~cm}^{-3}\right) \ln \Lambda T_{e}^{-1.5}(\mathrm{eV})$, we have:

$$
\tau_{/ /}=\left(\frac{2 L}{\pi}\right)^{2} \frac{2 \times 10^{-6} Z n_{e} \ln \Lambda}{v_{i}^{2} T_{e}^{1.5}(e V)}
$$

This $\tau_{/ /}$is to be compared with recent experiments.

Figure 1 shows our experimental 1/e-decay times measured for the Caprice $14 \mathrm{GHz}$ afterglow pulses as a function of the gas-fill pressure (p) with different rfpowers as a parameter. In this figure, the dependence of $\tau$ on the pressure (proportional to $\mathrm{n}_{\mathrm{e}}$ ) fits well by $1 / \mathrm{p}$ curves, and $\tau$ increases with the rf-power (proportional to $\mathrm{T}_{\mathrm{e}}$ ); completely opposite from what are predicted by Eq. 
(2). This implies that the parallel ambipolar diffusion is not adequate either, since $D_{a m b / /} \cong 2 D_{i}$.

Thus, the most of ions must be diffusing across $\mathrm{B}_{\mathrm{Z}^{-}}$ field. Coefficient of such a classical diffusion is given

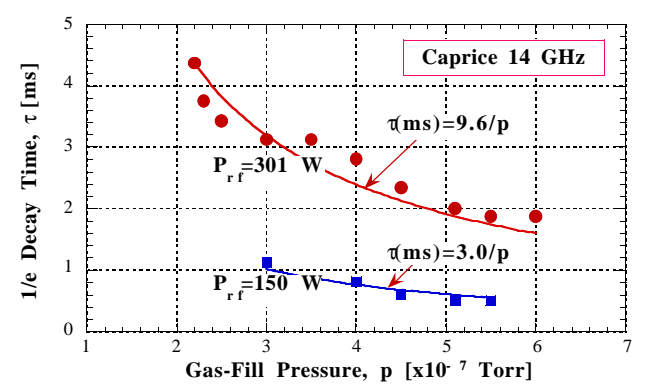

Figure 1: Pressure and rf-power dependence of decaytime.

by $\left.D_{i \perp} \equiv D_{i} / 1+\omega_{c i}^{2} \tau_{e i}^{2}\right) \cong 2 v_{e i}\left(v_{i} / \omega_{c i}\right)^{2}$, if $\omega_{c i} \tau_{e i} \gg 1$. Here, $\tau_{e i}=1 / v_{e i}$ and $\eta_{\perp}=2 \eta_{/ /}$were used. Relationship $\tau=(2 L / \pi)^{2} / D_{i \perp}$ gives the form to agree with Fig.1:

$$
\tau_{\perp}=\left(\frac{2 L}{\pi}\right)^{2}\left(\frac{\omega_{c e}}{v_{i}}\right)^{2}\left(\frac{m_{e}}{m_{i}}\right)^{2} \frac{Z T_{e}^{3 / 2}(e V)}{4.0 \times 10^{-6} n_{e} \ln \Lambda}
$$

However, Eq. (3) can not explain the $1 / \mathrm{Z}$ dependence shown by Fig. 2, where the data points are after Tinschert et al. [2]. During the afterglow the $\mathrm{B}_{\mathrm{z}}$-field configuration is intact, and hence the axial escape of rf-heated electrons is inhibited due to the magnetic mirrors. This means that most of ECR electrons ought to diffuse radially across $\mathrm{B}_{\mathrm{Z}^{-}}$ field together with ion flux. An ambipolar electric field, $\mathrm{E}_{\mathrm{amb}}$, is then induced for the radial direction. This $\mathrm{E}_{\mathrm{amb}}$ would not be short-circuited on the conductive endplates, since the electrons along the field lines would not reach there. Otherwise-slower-electron's cross-diffusion is accelerated by $E_{a m b}$ so that the flux can become equal with ion flux at the radial wall. Since the mobilities are obviously $\mu_{i \perp}>>\mu_{e \perp}$, and because $\mathrm{T}_{\mathrm{e}}>>\mathrm{T}_{\mathrm{i}}$ for all ECRIS plasmas, we have $D_{a m b \perp} \approx D_{e \perp}$. The form $\tau_{a m b \perp} \cong(2 L / \pi)^{2} / D_{e \perp}$ is still valid for our case because $\mathrm{n}_{\mathrm{e}}$ is the density of field particle, independent from that of test particle. Therefore, we have:

$$
\tau_{a m b \perp}=\left(\frac{2 L}{\pi}\right)^{2} \omega_{c e}^{2} \frac{\sqrt{T_{e}(e V)}}{7.0 \times 10^{5} Z n_{e} \ln \Lambda}
$$

This $\tau_{a m b \perp}$ agrees very well with all the experimental trends observed, including the numerical values. Figure 2 shows the two curves of Eq. (4) calculated for $\mathrm{T}_{\mathrm{e}}=100$ and $500 \mathrm{eV}$, when reasonable plasma parameters were assumed as shown in the inset. This may justify our previous conjecture: constant $D_{a m b \perp}$ or linear Eq. (1). The higher the $\mathrm{Z}$, the smaller the $\mathrm{L}$ for the HCI and it will experience a smaller $B_{Z}$ due to its shift toward the axis, which gives an extra decay in $\tau$ as expected by Eq. (4). This can explain the data points showing an excess decay at higher Z's in Fig. 2.

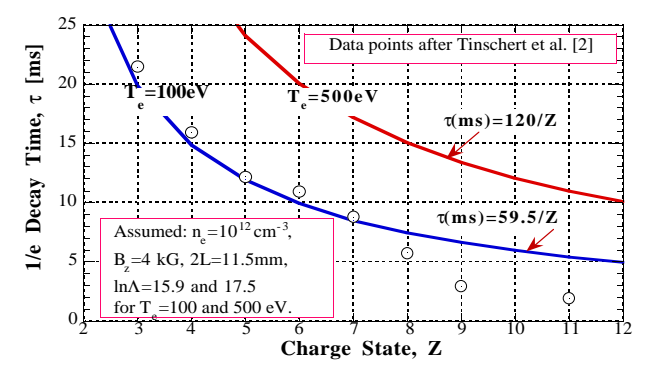

Figure 2: Z-dependence of $\tau$ and two simulated curves.

\subsection{Nonlinear Mode and Dynamics of $\Delta \phi$}

Beside the exponential decays of trailing edge an afterglow pulse depicts many different waveforms. They are found, including the leading edges, all belong to NLM, where the dynamics of $\Delta \phi$ plays an important role. Experimental data can be explained only if we assume that the density of hot (not the cold nor warm) electrons, $n_{h}$, is solely responsible for $\Delta \phi$. One can then write explicitly:

$$
\Delta \phi(V) \equiv \frac{n_{h} e}{4 \varepsilon_{o}} a^{2}=4.52 \times 10^{-9} n_{h}\left(m^{-3}\right) a^{2}(m)
$$

Equation (5) tells that either a loss of $n_{h}$ or a contraction of the radius "a" of $\mathrm{n}_{\mathrm{h}}$ lets the $\Delta \phi$ suddenly or slowly decrease like $\Delta \phi=\Delta \phi_{o} \rightarrow 0$, thereby amplifying $\mathrm{n}_{\mathrm{i}}$ (up to 10 times in our case) as $n_{i}=n_{c w} \rightarrow n_{p k}$ via the formula:

$$
n_{c w}=n_{p k} \exp \frac{-Z e \Delta \phi}{k T_{i}} \text { or } n_{p k}=n_{c w} \exp \frac{Z e \Delta \phi}{k T_{i}}
$$

This is shown in Fi. 3. Here, Type-A is the case of $\mathrm{n}_{\mathrm{c}} \geq \mathrm{n}_{\mathrm{h}}$, where $\Delta \phi$ decays immediately at $\mathrm{t}=\tau_{\text {off }}$ due to a sudden and complete loss of $n_{h}$ via interchange instability (ICI), while Type B is the case of $\mathrm{n}_{\mathrm{c}}>>\mathrm{n}_{\mathrm{h}}$, when $\Delta \phi$ decreases relatively slowly, prior to ICI, due to a slow contraction of "a," caused by a loss of the core pressure of cold electrons, $\mathrm{n}_{\mathrm{c}} \kappa \mathrm{T}_{\mathrm{c}}$ [See Eq. (8)].

Details on the relevant ICI have been studied elsewhere [3]. Sudden and almost complete loss in $n_{h}$ takes place if the ratio $\mathrm{n}_{\mathrm{h}} / \mathrm{n}_{\mathrm{c}}$ satisfies the instability (or ICI) criterion:

$$
\frac{n_{h}}{n} \cong \frac{n_{h}}{n_{c}} \geq \text { Const } \cong 0.15 m^{\prime} \frac{M}{Z}, \quad \text { if } \omega_{o} \cong \omega_{c i} \frac{M}{Z}
$$

In Figs. 3 and 4 , Const $=1 \quad\left(m^{\prime}=6,7\right)$ was assumed for simplicity. Here, $\omega_{0}$ is the angular frequency of hotelectron evolution, $m^{\prime}=1,2, \cdots, \quad$ and assumed $n=n_{h}+n_{c} \cong n_{c}$. Why then, like the case of Type-A in Fig. 3, an unstable plasma could be kept at equilibrium before $t=\tau_{\text {off }}$ ? In order to answer the question we propose the following pressure balance equation:

$$
n_{c} \kappa T_{c}+n_{h} m_{e} \frac{v_{h \theta}^{2}}{R_{b}}+F_{N L}=j_{\theta} \otimes B_{z}
$$

Here, $R_{b}$ is the radius of hot-electron evolution and $\mathrm{F}_{\mathrm{NL}}$ is the nonlinear force due to radiation pressure gradient, 
which can be significant for the device like ECRIS with a high-power microwave source. $\mathrm{F}_{\mathrm{NL}}$ is larger where the
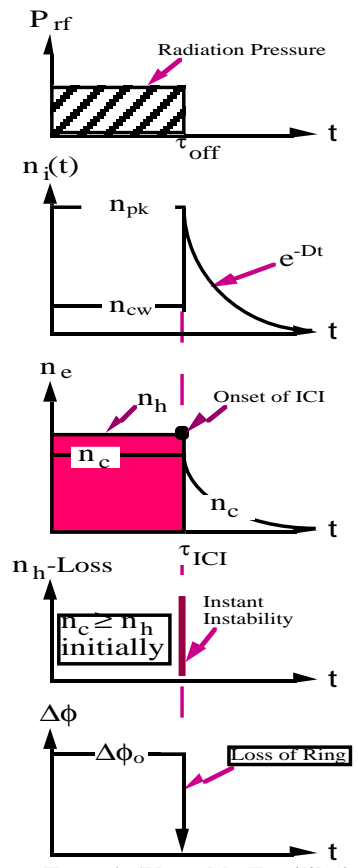

Type-A (Unstable Equilibrium)
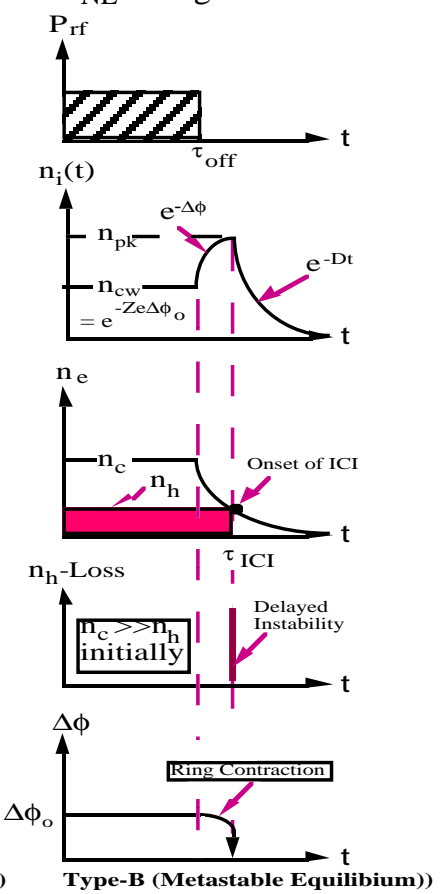

Figure 3: Diagram to explain real $n_{i}(t)$ from inelastic- $\Delta \phi$.

plasma density $\left(\sim \omega_{\mathrm{p}}\right)$ is higher, since

$$
F_{N L}=-\frac{\omega_{p}^{2}}{\omega_{r f}^{2}} \nabla \frac{<\varepsilon_{o} E^{2}>}{2}
$$

Therefore, as $\mathrm{F}_{\mathrm{NL}} \rightarrow 0$ at $\mathrm{t}=\tau_{\text {off }}$, a force-imbalance is manifested, which would generate a ripple to grow by ICI for the first time on the surface between plasma and vacuum magnetic field. Before $\mathrm{t}=\tau_{\text {off }}$, a perfectlyaxisymmetric radiation pressure of the microwave electric field, $E=E_{S} \exp \left(-j \omega_{\mathrm{rf}} \mathrm{t}\right)$, had kept the surface ripple free.

Very importantly, the experimental waveform of delayed instability as shown by Type-B in Fig. 3 can confirm that the instability to take place at the peak is nothing but ICI, since the step-like pulse-shape matches to the prediction by Eq. (7). Cold electrons are lost quickly after $\mathrm{t}=\tau_{\text {off }}$, having a large mirror loss and a faster diffusion loss as seen from Eqs. (3) and (4). Therefore, the initially-stable plasma $\left(\mathrm{n}_{\mathrm{c}}>>\mathrm{n}_{\mathrm{h}}\right)$ can become unstable after a certain time, when $n_{c}$ decreases to satisfy the ICI criterionof Eq. (7).

Now the presence of ICI has been proved. Then the centrifugal term $v_{h \theta}^{2} / R_{b}$ in Eq. (8) is real as an equivalent gravitational field. Thus we can conclude that the hot electrons are rotating by the velocity $v_{\mathrm{h} \theta}$ at the radius $a=R_{b}$ in the direction of diamagnetic current. Notice that this $R_{b}$ can contract (Fig. 3) and expand (Fig. 4) without a loss of $n_{h}$ before and after the onset of ICI, respectively. Contraction of $\mathrm{R}_{\mathrm{b}}$ is possible since the cold-electron pressure, $n_{c} \kappa T_{c}$ is perpetually lost from the core after $\mathrm{t}=\tau_{\text {off }}$. Expansion is possible because the constriction force, $j_{\theta} \otimes B_{z}$, where $j_{\theta} \equiv e n_{h} v_{\theta}$, decreases suddenly after a sudden loss of $n_{h}$. This situation can be seen in Fig. 4 .
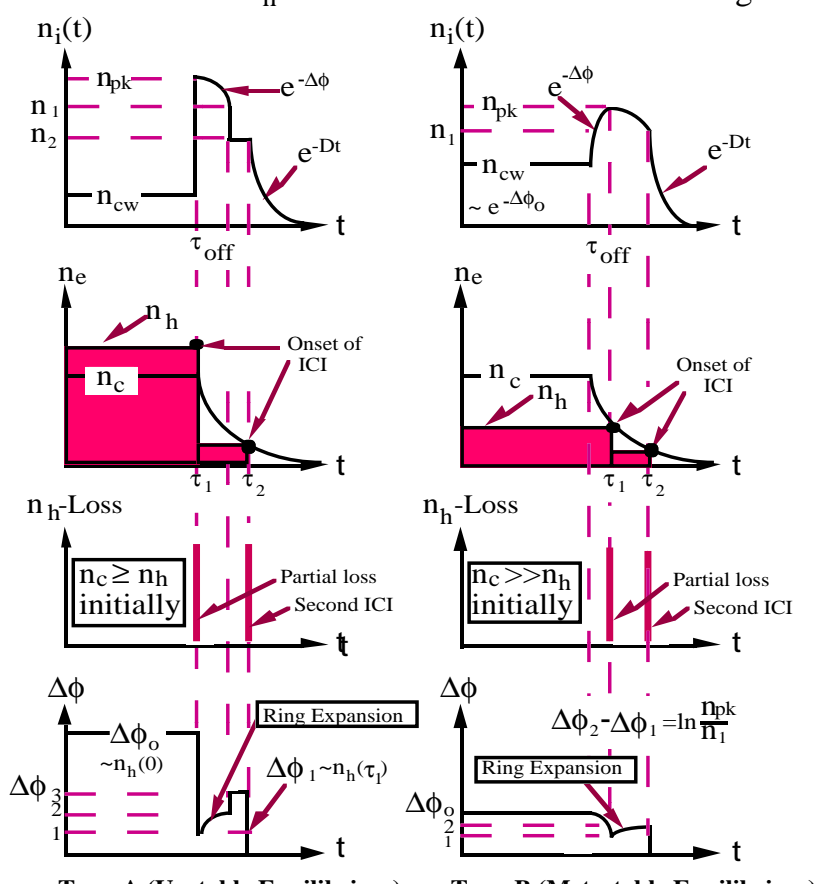

Type-A (Unstable Equilibrium)

Type-B (Metastable Equilibrium)

Figure 4: Diagram to explain real $n_{i}(t)$ from elastic- $\Delta \phi$.

If the loss of $n_{h}$ is total by ICI, the $\Delta \phi$ is inelastic: no oscillatory motion. But, if it is partial, the system is elastic. When another rf-pulse is applied within the life of hot-electrons left from the last rf-pulse, a so-called "2nd (ion) pulse" can be generated, since $\Delta \phi \neq 0$ if $\mathrm{n}_{\mathrm{h}} \neq 0$. Criterion of Eq. (7) is then immediately met since $n_{h}$ increases quickly with rf-power, while the level of $n_{c}$ is low. Cold electrons are slow to be confined inside the ECR zone. Thus, a pulse like Type-A in Fig. 3 is generated in the beginning $(\mathrm{t}=0)$ of rf-pulse, as in Fig. 5 .

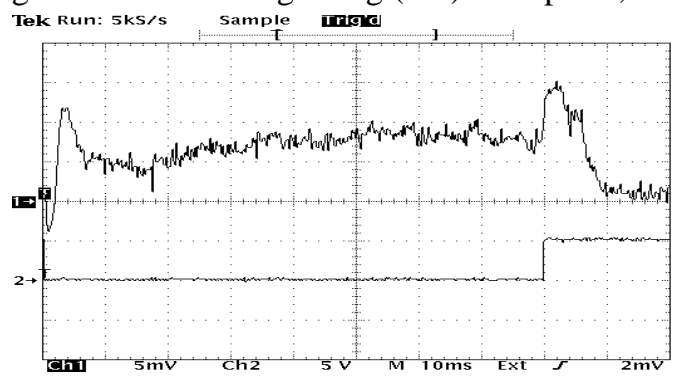

gian 2 ooo

Figure 5: Step-like afterglow pulse and a 2nd pulse at $\mathrm{t}=0$.

\section{ACKNOWLEDGEMENT}

One of the authors, MN, would like to acknowledge the support by National Research Council, National Academy of Science of the USA. This work was carried out in part at JPL/Caltech, and supported through NASA.

\section{REFERENCES}

[1] R. Geller, Electron Cyclotron Resonance Ion Sources and ECR Plasma, IOP Publishing, Inc., 1996; p. 257.

[2] K. Tinschert et al., Proceedings of ECRIS 99, p.102.

[3] M. Niimura et al., Rev. Sci. Instru. 71, 846 (2000). 